\title{
Precise shape grading of coarse aggregate
}

\author{
L. J. O'Flannery* and M. M. O’Mahony† \\ Lee MCullough and Partners; Trinity College Dublin
}

\begin{abstract}
The objective of the research described here was to derive dimensional parameters to provide a unique descriptive fingerprint for any sample of coarse-aggregate particles. The practical component of the work focused on devising a quick and accurate field test capable of yielding values for the descriptive parameters to enable a coarseaggregate sample to be quantitatively appraised. The aim of the paper is to propose this test as a means of ensuring quality control of coarse-aggregate shape. The parameters used were derived from first principles and applied to realistic particle shapes using simple mathematical models. Standard specifications were analysed to identify finite limits of acceptance for specific applications. These limits were then converted into precise terms of reference for the parameters. Extensive laboratory tests were performed on coarse-aggregate samples using the innovative test regime. The results show that such a sistem can be useful in providing information about an aggregate.
\end{abstract}

KEYWORDS: coarse aggregate; shape; grading

\section{Notation}

$I_{c} \quad$ cubicity index

$I_{\mathrm{r}} \quad$ roundness index

$I_{\Sigma} \quad$ sphericity index

$R_{1} \quad$ ratio of $Y$ dimension to $X$ dimension

$R_{z} \quad$ ratio of $Z$ dimension to $X$ dimension

$t \quad$ tolerance

$r_{\mathrm{a}} \quad$ volume of aggregate

$r_{\mathrm{C}} \quad$ volume of ellipsoid

$V_{\mathrm{o}} \quad$ volume of obloid

$W_{\mathrm{a}} \quad$ mass of aggregate in saturated surface-dry (SSD) condition

$\gamma \quad$ specific gravity

\section{Introduction}

Coarse aggregates are a fundamental component in many fields of construction throughout the world, for

\footnotetext{
$\doteqdot$ Lecturer, Department of Civil, Structural and Environmental Engineering. Trinity College Dublin, Ireland.

* Engineer. Lee. MCullough and Partners, formerly student, Trinity College. Dublin, Ireland.
}

(MCR 723) Paper received 7 September 1998; last revised 5 January 1999: accepted 3 March 1999. example concrete (mass and reinforced), roadworks, backfill, drainage and sewage treatment. There are several key factors that influence the ultimate suitability of an aggregate for a particular application, of which compressive strength, shape and texture are the most important. The objective of the proposed work is precisely to classify the shape of any coarse aggregate by means of three new indices. The cubicity index $I_{\mathrm{c}}$ will be used to describe the overall shape of the particle, and the sphericity index $I_{\mathrm{s}}$ and the roundness index $I_{\mathrm{r}}$ will be used to indicate the effective surface curvature of the particle.

The shape requirements, on a macroscopic scale, for aggregates for use in concrete, are essentially such that the particles produce a dense, interlocked mass. If the resultant mass is of less than optimum density, the finished concrete will rely too heavily on the sand cement matrix for load transfer and will be accordingly weaker than a concrete that transmits imposed loads through the coarse-aggregate structure. John ${ }^{1}$ found that perfectly rounded particles may compact with a porosity of approximately $48 \%$ when ordered in openpack formation, whereas De Wiest ${ }^{2}$ found more angular particles, similarly ordered, will tend to exhibit a porosity of up to $60 \%$. Other tests ${ }^{1}$ found porosity values of $26 \%$ for perfectly rounded particles of varying diameters in close-pack formation, whereas De Wiest ${ }^{2}$ measured porosity values of $0 \%$ for angular particles such as cubes. In tests with pentahedra and tetrahedra, 
it has been determined that the porosity may decrease below the $26 \%$ value mentioned above by virtue of interdigitation, i.e. impinging angular corners occupying interstitial space at an increasingly efficient rate.

The theoretical minimum porosity of $0 \%$ occurs when perfectly regular obloidal particles are arranged in an ideal formation. The minimum value is essentially ideal because it takes into account neither the possibility of non-obloidal shapes, nor the possibility of irregularity of shape or the random element of particle arrangement. The theoretical maximum porosity is slightly more complex since it must take into account the various possible particle shapes and arrangements. De Wiest ${ }^{2}$ quotes a value of $60 \%$, although he does not indicate the means by which this was found. However, a reasonable approximation may be derived from first principles.

In general, the requirements for aggregate shape are largely dependent on whether the resultant concrete element is required to withstand high or low stresses. In the former case the particles must be densely compacted and well interlocked. The particles must therefore be angular enough to compact well through interdigitation, but not too angular so as to produce excessive voids or risk breakage of the sharp corners. On the other hand, a concrete element expected to resist low levels of stress may be composed of either subangular, subrounded or rounded coarse-aggregate particles.

\section{Theoretical shape requirements}

Lees ${ }^{3}$ states that the shape characteristics of aggregates can have marked effects on the properties of both fresh and hardened concrete. These effects tend to be beneficial where the predominant particle shape is generally equidimensional, and detrimental where it is flaky and/or elongated. ${ }^{4-6}$ A certain amount of flakiness and elongation is generally tolerated, although it would seem more meaningful to convert the specified percentage of cubical and non-cubical shapes into a single measure of shape with which the average shape of the particles could be compared. Such a method is proposed below.

Let the longest, middle and shortest dimensions of a particle be termed $X, Y$ and $Z$, respectively. Let each dimension be represented as a proportion of the longest dimension by dividing each by $X$, and let the proportions of $Y$ to $X$ and $Z$ to $X$ be termed $R_{y}$ and $R_{z}$, respectively. This method serves to simplify the description of the aggregate particle's shape in broad terms, by describing proportions of a bounding obloid. Employing this method to a particle which has dimensions $20 \mathrm{~mm}, 15 \mathrm{~mm}$ and $10 \mathrm{~mm}$, yields, for example, the descriptive parameters $R_{y}=0.75$ and $R_{z}=0.5$. Typical standards for concrete suggest that the descriptive parameters $R_{y}$ and $R_{z}$ must lie within the range 0.6 to $1 \cdot 00$. Therefore one could consider that a typical cubical particle would have parameters of $R_{y}=0.8$ and
$R_{z}=0 \cdot 8$. The differences from the extremes of the range are $\pm 0 \cdot 2$, giving a tolerance of $\pm 25 \%$. A typical non-cubical particle may be similarly expressed by $R_{y}=0.3$ and $R_{z}=0.3$, with a tolerance of $\pm 100 \%$.

ASTM C $33^{7}$ does not place a limit on the percentage of non-cubical particles allowed but other standards do. For example, the Irish Standard IS $5^{8}$ suggests that the maximum percentage of non-cubical particles permitted in structural concrete is $40 \%$. Using $40 \%$ and the previously derived descriptive parameters as an example, it would be valid to derive a description of the desired mean particle as $60 \%$ cubical and $40 \%$ non-cubical, where $R_{y}=0.6 \times 0.8+0.4 \times 0.3=0.6$ and where, similarly, $R_{z}=0.6$. The tolerance may be calculated in a similar manner as $t=0.6 \times 25+0.4 \times 100=55 \%$. Applying the tolerance to the desired mean yields a maximum value of $R_{y}$ and $R_{z}$ of $0.6 \times(1.00+$ $0.55)=0.93$. Similarly, the minimum value of $R_{y}$ and $R_{z}$ can be calculated to be 0.27 .

Compliance with the IS $5^{8}$ standard is achieved when the mean values for $R_{y}$ and $R_{z}$ are not less than 0.6 and individual values for $R_{y}$ and $R_{z}$ are not less than 0.27 . Applying these constraints to a nominal $20 \mathrm{~mm}$ particle with an $X$ dimension equal to $20 \mathrm{~mm}$, the mean $Y$ and $Z$ values for the sample must not be less than 12.0 and the minimum value for any individual $Y$ or $Z$ value is $5.4 \mathrm{~mm}$. Since the assumed accuracy of compliance with the standard tests (BS $\left.812^{9}\right)$ is $95 \%$ confidence, it would seem reasonable to consider the mean $R_{y}$ and $R_{z}$ values of $0.6 \times 0.95=0.57$ as borderline but acceptable, i.e. the mean $Y$ or $Z$ value of $12.0 \mathrm{~mm}$ could decrease to $11.4 \mathrm{~mm}$ and still satisfy the specified requirements.

\section{Aggregate production}

Holmes ${ }^{10}$ proposed two general properties common to all types of rock that provide the basis for strength and durability, namely crystalline interlock and crystalline bonding. Crystalline interlock is defined as the tendency of the individual particles to impinge on each other because of their interwoven orientation, also known on a macroscale as interdigitation. Crystalline bonding is defined as the chemical binding forces that induce the crystals to adhere to one another through the cementing action of a pervading matrix. Robinson ${ }^{11}$ stated that igneous rocks possess a strongly defined crystalline structure with relatively large crystals which rely solely on immensely powerful crystalline interlock for strength. The influence of interlock in sedimentary rocks is far less apparent because the rounded, cemented particles lack crystalline angularity, with a resultant decrease in strength. Metamorphic rocks are generally the most dense, being formed under greater forces than the other two types. ${ }^{10}$ These rocks possess strength due to crystalline interlock but also planes of weakness from degraded crystalline bonding because of mechanical and chemical alteration.

Magazine of Concrete Research, 1999, 51, No. 5 
Coarse-aggregate particles are commonly formed by natural and/or induced breakage of the parent rock into polyhedral shapes, which may then be further modified by rounding. The original polyhedral shape is largely dictated by the type and condition of the parent rock and the method of breakage. There are three distinct polyhedral types for the purposes of engineering: tetrahedra, having four faces; pentahedra, five faces; and hexahedra, six faces. For practical purposes, particles with more than six faces may be considered to be hexahedral with rounding.

There are two broad categories of coarse aggregates, which generally reflect both their origin and their overall shape, i.e. crushed and uncrushed. Crushed aggregates are formed by passing large pieces of rock through a configuration of crushers, which invariably results in producing aggregate with a high degree of angularity. Cncrushed aggregates are naturally occurring pebbles found in coastal areas, along river beds and in glacial moraines.

\section{Indices}

It is mathematically convenient to consider, as a general case, an aggregate particle that is a slightly rounded. regular hexahedron proportioned somewhere between an obloid (three-dimensional oblong) and an ellipsoid (three-dimensional ellipse). As a convention, the longest dimension of the particle will be termed $X$ in the $x$ direction, the vertical dimension will be termed $Y$ in the $y$ direction and the third dimension will be termed $Z$ in the $z$ direction.

The first property for consideration is form. Consider a particle as a perfect obloid of dimensions $X, Y$ and $Z$. This obloid may be said to be the bounding obloid of the true particle since it shares the same overall dimensions. The concept of cubicity is now introduced. Let the cubicity index $\left(I_{\mathrm{C}}\right)$ be defined as the volume of the obloid expressed as a percentage of a bounding cube. The cube will possess equal $X, Y$ and $Z$ dimensions:

$$
I_{\mathrm{c}}=\frac{100 X Y Z}{X^{3}}
$$

The cubicity index provides immediate indications of the likely degree of potential compaction and therefore the probable porosity, subject to the particle's ability to integrate with neighbouring particles by interdigitation and rounding.

When dealing with a more realistic case of a particle shaped as a slightly rounded regular hexahedron, the true volume of the particle will normally lie between the volume of the bounding obloid and the volume of the ellipsoid of identical principal dimensions. The volume of the obloid is given as

$$
V_{\mathrm{o}}=X Y Z
$$

and the volume of the ellipsoid is

Magazine of Concrete Research, 1999, 51, No. 5

$$
V_{\mathrm{e}}=\frac{\pi X Y Z}{6}
$$

The concept of sphericity is now introduced, where the sphericity index $\left(I_{\mathrm{s}}\right)$ is the volume of the aggregate expressed as a percentage of the bounding obloid. It may be described as

$$
I_{\mathrm{s}}=\frac{100 V_{\mathrm{a}}}{V_{\mathrm{o}}}
$$

where $V_{\mathrm{a}}$ is the volume of the aggregate. For the purposes of the paper the indices will be expressed as dimensionless integers.

\section{Particle shape}

Particles with an $I_{\mathrm{c}}$ value between 60 and 100 may be classed as cubic, whereas $I_{\mathrm{c}}$ values between 30 and 60 indicate flakiness and values below 30 suggest elongation. There is a broad distinction between hexahedra, with $I_{\mathrm{S}}$ values between 50 and 100 , and both pentahedra and tetrahedra, with $I_{\mathrm{s}}$ values between 17 and 50 , where tetrahedra have values close to the former and pentahedra to the latter. The accurate quantification of angularity and roundness is more appropriately described by the value of the roundness index $I_{\mathrm{r}}$, where particles with $I_{\mathrm{r}}$ values between 84 and 100 may be classed as angular, while particles with $I_{\mathrm{r}}$ values between 52 and 68 may be classed as rounded. It is apparent from this method of description that terms such as subrounded and subangular may now be designated precise numerical values for determination by an objective and universal test.

In the case of a hexahedron, $I_{\mathrm{r}}=I_{\mathrm{s}}$. For a pentahedron, $I_{\mathrm{r}}=2 I_{\mathrm{s}}$ and in the case of a tetrahedron, $I_{\mathrm{r}}=6 I_{\mathrm{s}}$. The $I_{\mathrm{r}}$ values seldom falls below 50 unless the aggregate is prone to conchoidal or concave fracture. Relating porosity to these shape indices, it has been found that $\left(100-I_{5}\right)$ may be regarded as the maximum porosity of the aggregate since it represents the porosity associated with an ideal square packing arrangement.

The strongest advantage of describing an aggregate particle in terms of the index values mentioned above is that the definition is both precise and universally reproducible, regardless of rock type, condition or geologic origin. The second advantage is that it enables the aggregate shape to be assessed relatively quickly by means of a short and simple test which may be carried out in the field.

\section{Test for measurement of indices}

The principal objective of the test was to accurately measure and record physical dimensional data of samples of coarse-aggregate particles. The data was subsequently employed in calculations to provide values for 
$I_{\mathrm{c}}, I_{\mathrm{S}}$ and $I_{\mathrm{r}}$, which form a definitive and reliable means of describing the particle shape. Two samples, each containing 100 particles, were subdivided from a larger sample by random selection. Each particle in each sample was measured for $X, Y$ and $Z$ dimensions using a vernier caliper. The dimensions were recorded to the nearest millimetre and the particles were weighed to the nearest $0 \cdot 1 \mathrm{~g}$. From these dimensions the values of $R_{x}, R_{y}, R_{z}, V_{\mathrm{o}}$ and $I_{\mathrm{c}}$ were found. The $I_{\mathrm{S}}$ value was calculated using a measurement of the volume of the aggregate by means of the BS $812^{12}$ test or a field test version of the test using volumetric displacement. The latter tends to yield results within $3 \%$ of the former and takes $0.5 \mathrm{~h}$ to execute, compared with the BS test, which takes two days.

Having determined the specific gravity $(\gamma)$ for the saturated, surface-dried (SSD) sample, the actual volume $\left(V_{\mathrm{a}}\right)$ was calculated using the expression

$$
V_{\mathrm{a}}=\frac{W_{\mathrm{a}}}{\gamma}
$$

where $W_{\mathrm{a}}$ is the mass of the aggregate in an SSD condition. $I_{\mathrm{s}}$ was then calculated in accordance with equation (4).

As the analysis of the results assumes random values constrained by an $S$ distribution, according to Francis ${ }^{13}$ it was necessary for the sample size to exceed 30 in number as a prerequisite for probabilistic validity. The nominal aggregate sizes under consideration were $14 \mathrm{~mm}, 20 \mathrm{~mm}$ and $40 \mathrm{~mm}$. It was found to be convenient to use 100 particles of the $14 \mathrm{~mm}$ and $20 \mathrm{~mm}$ aggregates and 50 particles of the $40 \mathrm{~mm}$ aggregate. Initially two samples of each aggregate were tested, but as the results proved consistent a single sample was used thereafter.

At the same time as the principal dimensions of each particle were being measured and recorded, the shape of the particle was examined and recorded as an approximation of either a hexahedron, a pentahedron or a tetrahedron. In the event that a definite form was not obvious, the approximation of hexahedral was awarded by default.

The maximum and probable errors associated with the test will now be considered. The first phase of the test was the determination of the dimensions. The error in this phase is associated with the rounding up of each particle dimension to the nearest millimetre, giving a resultant error of $\pm 0.5 \mathrm{~mm}$ and a probable error of $\pm 0.25 \mathrm{~mm}$. It is useful to express the maximum possible error in terms of a percentage of the total volume of the bounding obloid, which in turn is a function of both the nominal particle size and the dimensional regularity. If the error is expressed as a percentage of the $I_{c}$ value the maximum error for a $20 \mathrm{~mm}$ nominal particle, for example, will be $\pm 7.5 \%$.

After the shape parameters of the aggregate were established, the porosity of the bulk aggregate was measured using a test similar to the voids measurement test in BS $812 .^{12}$

\section{Test results}

Four types of aggregate, described as follows, were tested using the proposed regime:

- type A: $14 \mathrm{~mm}, 20 \mathrm{~mm}$ and $40 \mathrm{~mm}$ nominal sizes of crushed basaltic andesite

- type B: $14 \mathrm{~mm}, 20 \mathrm{~mm}$ and $40 \mathrm{~mm}$ nominal sizes of crushed siliceous limestone

- type C: $14 \mathrm{~mm}$ and $20 \mathrm{~mm}$ nominal sizes of crushed greywacke

- type D: $14 \mathrm{~mm}, 20 \mathrm{~mm}$ and $40 \mathrm{~mm}$ nominal sizes of partially crushed limestone gravel.

Each test sample was coded to indicate the parent source, nominal particle size and identity. For example, the two test samples of $14 \mathrm{~mm}$ greywacke were coded $\mathrm{C} 1 \mathrm{~A}$ and $\mathrm{C} 1 \mathrm{~B}$, where ' $\mathrm{C}$ ' means type $\mathrm{C}$, ' 1 ' is for $14 \mathrm{~mm}$ nominal size and ' $\mathrm{A}$ ' and ' $\mathrm{B}$ ' refer to the sample number.

The specific gravity of the aggregates on an SSD basis ranged from 2.57 to 2.74 and the water absorption values on an oven-dried basis were $2 \cdot 5-2 \cdot 7 \%$. As expected, the results were quite consistent. Fig. 1 shows the porosity test results, where it can be seen that they fall within the range $41-58 \%$.

In Fig. 2 the field test results for $I_{\mathrm{c}}$ can be seen. In almost all cases the $I_{\mathrm{c}}$ values are between 30 and 60 , indicating a degree of flakiness in all samples. The standard deviations are presented in Table 1. In summary, they are relatively large, reflecting the natural variation of the material.

Figure 2 also shows the field test results for $I_{\mathrm{s}}$, where it can be seen that the results are below 52, indicating for the most part that the particles show characteristics normally associated with pentahedra. The $I_{\mathrm{r}}$ results are also presented in Fig. 2. When these results are examined in detail (Table 1) it can be seen

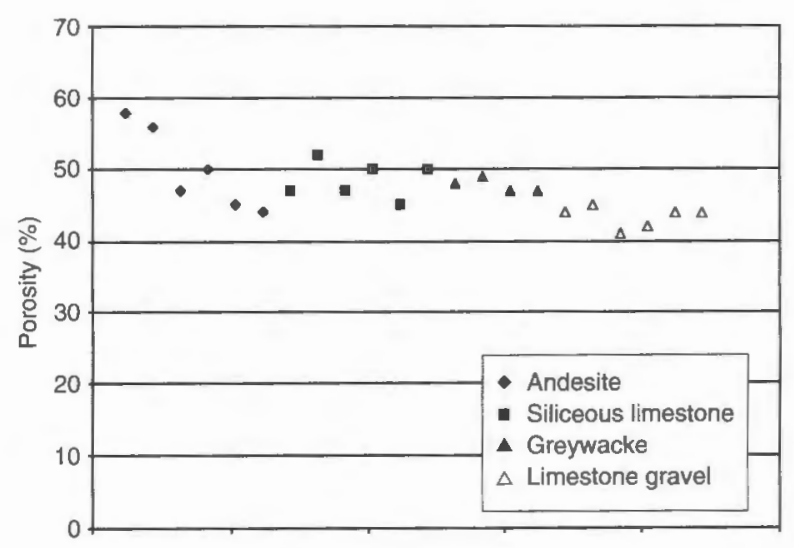

Fig. I. Porosity results

Magazine of Concrete Research, 1999, 51, No. 5 


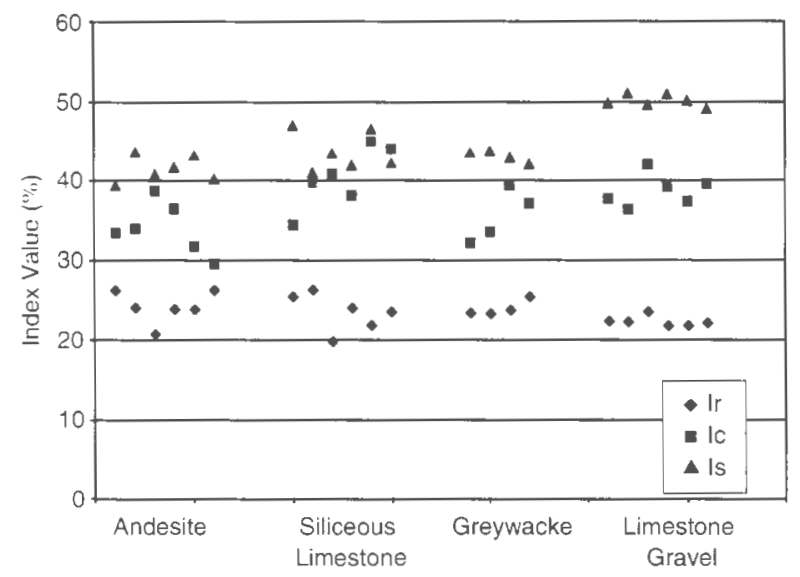

Fig. 2. Indices of samples

that the smaller particle sizes, i.e. $14 \mathrm{~mm}$ and $20 \mathrm{~mm}$, of the basaltic andesite and partially crushed limestone gravel are angular. whereas the $40 \mathrm{~mm}$ particles are rounded. This may be due to the higher levels of crushing required for smaller particle sizes. The crushed siliceous limestone follows this trend in part but the conclusion is not as convincing in this case. The $20 \mathrm{~mm}$ crushed greywacke particles indicate rounding, whereas the $1+\mathrm{mm}$ particles are angular.

Figure 3 shows the test results for the shape profiles of the samples. expressed in percentages of tetrahedral. pentahedral and hexahedral particles. There is good correlation between profiles for pairs of samples, despite the relatively small sample size and the variability of the material.

When comparing the results with the standards for concrete. it can be observed that ten out of 22 samples

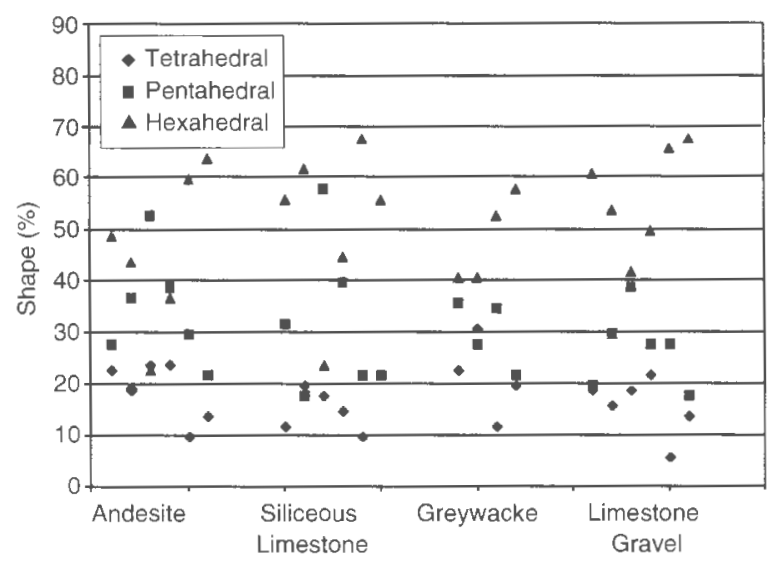

Fig. 3. Results for shape profiles

(45\%) fail to provide $95 \%$ confidence in the smallest dimension and may be judged flaky by BS $812^{9}$ for concrete production. Having a means of identifying such an issue may help concrete designers in their choice or combination of materials.

\section{Classification by test parameters}

The test yields values for three parameters that are unique to the sample and may be used as a description or 'fingerprint' for the coarse-aggregate particles. It was originally envisaged that simple mathematical modelling would be able to entirely replace the present empirical method of visual inspection and classification into the categories of round, irregular and angular. However, it has been found that while an expression

Table 1. Measurements of indices

\begin{tabular}{|c|c|c|c|c|c|c|c|}
\hline Sample ID & $\begin{array}{c}\text { Nominal size: } \\
\mathrm{mm}\end{array}$ & $I_{\mathrm{c}}$ & $\mathrm{SD}, I_{\mathrm{c}}$ & $I_{\mathrm{s}}$ & $\mathrm{SD}, I_{\mathrm{s}}$ & $I_{\mathrm{r}}$ & $\mathrm{SD}, I_{\mathrm{r}}$ \\
\hline$A 1 A$ & 14 & $33 \cdot 6$ & $14 \cdot 9$ & $39 \cdot 5$ & 6.9 & $66 \cdot 1$ & $26 \cdot 4$ \\
\hline AlB & 14 & $34 \cdot 1$ & $18 \cdot 2$ & $43 \cdot 7$ & 8 & $71 \cdot 3$ & $24 \cdot 2$ \\
\hline$A 2 A$ & 20 & $38 \cdot 8$ & $15 \cdot 5$ & $40 \cdot 9$ & - & $76 \cdot 8$ & $20 \cdot 9$ \\
\hline$A 2 B$ & 20 & $36 \cdot 6$ & $14 \cdot 2$ & $41 \cdot 8$ & - & 72 & 24 \\
\hline$A B A$ & 40 & 31.9 & 12.9 & $43 \cdot 3$ & $8 \cdot 2$ & $62 \cdot 8$ & 24 \\
\hline $\mathrm{A} 3 \mathrm{~B}$ & 40 & $29 \cdot 7$ & $10 \cdot 5$ & $40 \cdot 3$ & $6 \cdot 7$ & $58 \cdot 7$ & $26 \cdot 4$ \\
\hline B1A & 14 & $34 \cdot 6$ & 17 & $47 \cdot 2$ & $9 \cdot 6$ & $68 \cdot 5$ & $25 \cdot 6$ \\
\hline B1B & 14 & $39 \cdot 9$ & 17 & $41 \cdot 1$ & $8 \cdot 3$ & $62 \cdot 1$ & $26 \cdot 5$ \\
\hline $\mathrm{B} 2 \mathrm{~A}$ & 20 & $40 \cdot 9$ & $16 \cdot 9$ & $43 \cdot 6$ & 8.4 & $79 \cdot 8$ & 20 \\
\hline $\mathrm{B} 2 \mathrm{~B}$ & 20 & $38 \cdot 2$ & $15 \cdot 4$ & 42 & 7.0 & 68.7 & $24 \cdot 2$ \\
\hline B3.A & 40 & $45 \cdot 1$ & $16 \cdot 4$ & $46 \cdot 7$ & 8.6 & $62 \cdot 4$ & 22 \\
\hline B3B & 40 & $44 \cdot 1$ & $13 \cdot 6$ & $42 \cdot 3$ & $7 \cdot 7$ & 65.5 & $23 \cdot 7$ \\
\hline CIA & 14 & $32 \cdot 3$ & $14 \cdot 7$ & $43 \cdot 7$ & $8 \cdot 4$ & $72 \cdot 9$ & $23 \cdot 5$ \\
\hline $\mathrm{C} 1 \mathrm{~B}$ & 14 & $33 \cdot 7$ & $13 \cdot 2$ & $43 \cdot 8$ & $9 \cdot 4$ & 75 & $23 \cdot 5$ \\
\hline$C 2 A$ & 20 & $39 \cdot 5$ & $14 \cdot 1$ & 43 & $6 \cdot 4$ & $65 \cdot 5$ & $23 \cdot 9$ \\
\hline $\mathrm{C} 2 \mathrm{~B}$ & 20 & $37 \cdot 2$ & 14.7 & $42 \cdot 2$ & $6 \cdot 8$ & 64 & $25 \cdot 6$ \\
\hline $\mathrm{D} 1 \mathrm{~A}$ & 14 & $37 \cdot 8$ & $16 \cdot 2$ & 50 & $10 \cdot 1$ & $70 \cdot 2$ & $22 \cdot 5$ \\
\hline D1B & 14 & $36 \cdot 5$ & 15.9 & $51 \cdot 2$ & $9 \cdot 8$ & $73 \cdot 3$ & $22 \cdot 5$ \\
\hline $\mathrm{D} 2 \mathrm{~A}$ & 20 & $42 \cdot 1$ & 15 & 49.8 & $7 \cdot 4$ & $77 \cdot 3$ & $23 \cdot 7$ \\
\hline $\mathrm{D} 2 \mathrm{~B}$ & 20 & $39 \cdot 3$ & 14.9 & $51 \cdot 1$ & $8 \cdot 3$ & 75.6 & 22 \\
\hline D3A & 40 & 37.5 & 14.5 & $50 \cdot 3$ & $6 \cdot 8$ & 66.4 & 22 \\
\hline D3B & 40 & $39 \cdot 7$ & $15 \cdot 7$ & $49 \cdot 3$ & $6 \cdot 3$ & $65 \cdot 3$ & $22 \cdot 3$ \\
\hline
\end{tabular}


Table 2. A typical shape classification

\begin{tabular}{l|l}
\hline Shape type & Subangular \\
\hline Nominal size & $20 \mathrm{~mm}$ \\
\hline Shape profile & $20 \%$ tetrahedral \\
& $22 \%$ pentahedral \\
& $58 \%$ hexahedral \\
\hline
\end{tabular}

\begin{tabular}{l|c|c|c}
\hline Index & Value & SD & Coefficient of variation \\
\hline$I_{\mathrm{c}}$ & $37 \cdot 2$ & $14 \cdot 7$ & $39 \cdot 5$ \\
\hline$I_{\mathrm{s}}$ & $42 \cdot 2$ & 6.8 & $16 \cdot 1$ \\
\hline$I_{\mathrm{r}}$ & $64 \cdot 0$ & 25.6 & 40 \\
\hline$R_{x}$ & $1 \cdot 3$ & 0.24 & 18.5 \\
\hline$R_{y}$ & 0.72 & 0.14 & $19 \cdot 4$ \\
\hline$R_{z}$ & 0.50 & 0.13 & 26.0 \\
\hline
\end{tabular}

may be formulated to describe the proximity of a particle to an ideally rounded or angular model, it cannot discern whether the loss of protruding corners has resulted in angular or rounded surfaces. While the test is able to provide precise and accurate quantification of the particle shape, it is unable to make the fundamental distinction between the true roundness of a well-worn beach pebble and the effective roundness of a severely angular polyhedron of the same gross shape and volume. Consequently, it should be the role of the shape test to supplement rather than supplant the visual test. From the foregoing, it is apparent that a typical shape classification for a sample of coarse-aggregate particles would be similar to that presented in Table 2 .

\section{Conclusions}

The objective of the research was to devise a means of providing a precise, quantified description of a sample of coarse-aggregate particles. The method proposed was by the use of a set of shape indices. The paper defines a set of descriptive parameters for coarse aggregates and describes a test for finding them. It also discusses the results of the test on several coarse-aggregate samples and comments on its usefulness but also on its shortcomings. The method proposed in the paper is more quantifiable than other, more subjective method of describing particle shape. It effectively enables the determination of a 'fingerprint' for any coarse-aggregate sample.

\section{References}

1. JoHN V. B. Introduction to Engineering Materials. MacMillan, London, 1978.

2. De Wiest R. J. M. Geohydrology. Wiley, London, 1965.

3. LeEs G. The measurement of particle shape and its influence in engineering materials. Journal of the British Granite and Whinstone Federation, 1964, 4, No. 2.

4. KAPLAN M. F. The effects of the properties of coarse aggregates on the workability of concrete. Magazine of Concrete Research, 1958, 10, No. 29, 63-74.

5. KAPLAN M. F. Flexural and compressive strength of concrete as affected by the properties of coarse aggregates. Journal of the American Concrete Institution, 1959, 55, 1193-1208.

6. Neville A. M. Properties of Concrete, 3rd edn. Pitman, London, 1981.

7. AMERICAN SOCIETY for TESTING AND MATERIALs. Standard Specification for Concrete Aggregates. ASTM, Philadelphia, 1997, C33-97.

8. Aggregates for Concrete. Institution for Industrial Research and Standards, Dublin, 1974.

9. British Standardos lnstitution. Methods for Determination of Particle Shape. BSI, Milton Keynes, 1989, BS 812: Part 105.

10. Holmes A. Principles of Physical Geology. Nelson, Walton-onThames, 1978.

11. Robinson E. S. Basic Physical Geology. Wiley, New York, 1982.

12. British Standards InStitution. Testing Aggregates. BSI, Milton Keynes, 1995, BS 812: Part 2.

13. Francis A. Business Mathematics and Statistics. DP Publications, London, 1988.

Discussion contributions on this paper should reach the editor by 31 March 2000 